\title{
Nonequilibrium noise and instabilities in membranes with active pumps
}

\author{
SRIRAM RAMASWAMY, JOHN TONER* and JACQUES PROST ${ }^{\dagger}$ \\ Centre for Condensed Matter Theory, Department of Physics, Indian Institute of Science, \\ Bangalore 560 012, India \\ * Department of Physics, University of Oregon, Eugene OR 97408-1274, USA \\ † Institut Curie, Section de Recherche, 26 rue d’Ulm, 75231 Paris cedex 05, France
}

\begin{abstract}
We study the statistical properties and dynamics of flexible fluid membranes containing active transmembrane proteins and find that such active membranes can be either stable or unstable to small disturbances, depending on the signs of certain crucial parameters characterising the protein-membrane interaction. A major finding is that the shape-fluctuation spectrum in the stable case for a zero-tension active membrane has the same form over a large range of length scales as at equilibrium, but with a nonequilibrium "noise temperature" determined by the activity.
\end{abstract}

Keywords. Nonequilibrium noise; membranes; active pumps.

PACS Nos 05.40.-a; 87.16.Dg

\section{Introduction and results}

\subsection{Motivation}

The living cell $[1,2]$ is a system far from equilibrium, maintained as such by a variety of active energy-dissipating processes. These often involve the directed transport of substances across the cell wall or through internal boundaries, facilitated by specific proteins confined to the membrane that constitutes these boundaries. When such a protein is held in its active state (say by an imposed electrochemical gradient, as in ion channels, or the uptake of ATP or light energy, as in molecular pumps), it transfers ions and solvent across membrane in a specified direction. In doing so it exerts a force $F_{a} \sim T / w$ along the local normal to the membrane, where $T$ and $w$ are respectively the temperature and a molecular length. The force always acts towards a specific end of a given protein molecule, which we shall call its head (and the other end its tail). Figure 1 shows schematically a membrane containing a collection of such proteins. The filled (empty) circles have their heads (tails) up.

Despite the ubiquity of such active, nonequilibrium processes in biological membranes, the attention of physicists has largely been restricted to the statistical mechanics of membranes at thermal equilibrium [3,4]. Although such an approach has been very succesful in describing the shapes of vesicles and their statistics, there is growing evidence that there is more $[5,6]$ to the flicker of red blood cells than is contained in the equilibrium analysis of [7]. The first theoretical studies of "active membranes", i.e., 


\section{Sriram Ramaswamy, John Toner and Jacques Prost}

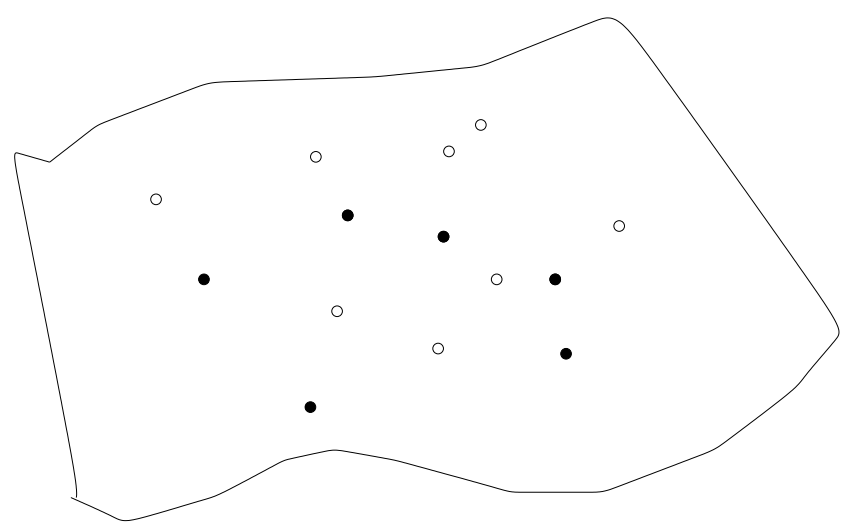

Figure 1. A membrane with pumps. Black circles: heads up; white circles: heads down.

membranes containing energised active proteins, predict striking nonequilibrium enhancements of fluctuations [8] and remarkable, fluctuation-induced long-range interactions [9]. Perhaps most dramatic are recent micropipette experiments on membranes laden with the photoactive proton pump bacteriorhodopsin (BR). When the BR is activated, the fluctuations have a form superficially identical to that at equilibrium, but with a magnitude much larger than can be accounted for by the thermodynamic temperature of the system [10].

\subsection{Summary of model and results}

In this paper, we consider the statistical mechanics and dynamics of a fluid membrane containing a distribution of identical, active proteins (hereafter often called "pumps") free to move in the plane of the membrane (see figure 1). We shall call a protein a "+ pump" ("- pump") if the vector from its tail to its head points parallel to (antiparallel to) a fixed outward normal of the membrane. We forbid flips from + to - , which are prohibitively slow in any real system. We shall call a membrane "balanced" or "unbalanced" according to whether the numbers of + and - pumps are equal or unequal. It is crucial to note that the functional asymmetry of the protein (unidirectional pumping) implies a structural asymmetry as well [11]: the "head" will in general be larger or smaller than the "tail", and the protein will therefore favour one sign of the membrane mean-curvature over the other (see figure 2). Gradients of the local mean curvature will thus give rise to motions of the pumps in the plane of the membrane.

Our main results, obtained largely within a linearised description of the membrane dynamics, are as follows: (i) The flat, homogeneous state of an active membrane can be either linearly stable or linearly unstable [12], depending on the signs of certain parameters arising only in the active state, and related to the structural and functional asymmetry of the protein. (ii) In the linearly stable case, an active membrane is in a nonequilibrium steady state with statistical properties which can mimic those of a membrane at thermal equilibrium. Specifically, for a "balanced" active membrane with zero tension, we show that the height variance over a wide range of wavenumbers $q$ is $\sim 1 / q^{4}$, but with a coefficient independent of the bending rigidity, inversely proportional to the transverse 


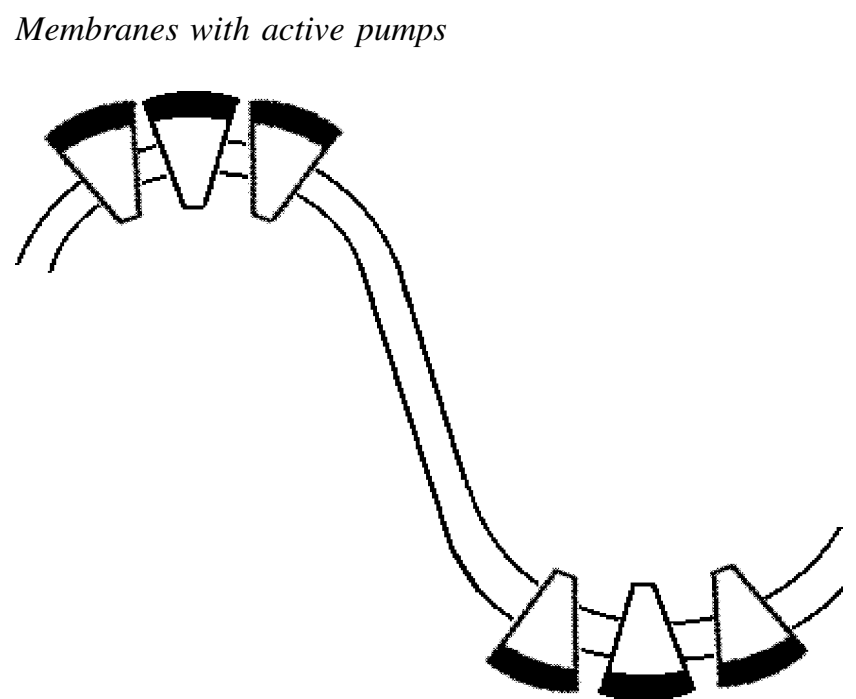

Figure 2. Side view of a membrane with active proteins. The asymmetric shape of the pumps means the pump can distinguish positive and negative mean curvature. This causes a pump to prefer regions in which the curvature is adapted to its shape, as seen in the picture. In addition (S Ramaswamy, J Toner, and J Prost, unpublished), it allows the pump activity to depend on the local mean curvature.

mobility of the protein and proportional to the protein activity. These features testify to the truly nonequilibrium origins of the fluctuations, which arise from variations in the local normal force caused by the Brownian motion of the pumps. The effect, in remarkable agreement with the observations of [10], is independent of the concentration of pumps. (iii) More detailed results on the statistical and dynamical properties of active membranes will appear elsewhere [14]. These include: (a) the occurrence of propagative modes with a speed independent of the membrane elasticity; (b) the ultimate fate of the linear instabilities (ordered patterns, coarsening, or spatiotemporal chaos) [15, 16]; and (c) nontrivial scaling exponents when nonlinearities are taken into account in the stable noise-driven case.

\section{The model}

\subsection{Simplified dynamical model for a balanced membrane}

The model within which these results were derived is a natural extension of the classic models of the dynamics of membranes at equilibrium [7]. To avoid complications associated with the hydrodynamic interaction, we shall assume in this paper that the membrane motion is damped locally by a frictional force proportional to its velocity. Our major results will largely be unchanged if this "Rouse"-like description (by analogy with polymer dynamics [17]) is replaced by a more realistic damping arising from coupling to a hydrodynamic velocity field as in [7]. The Rouse description is in any case appropriate for a membrane near a wall [9], on length scales large enough for permeation to be important but small enough that wandering-induced collisions with the wall can be neglected. We shall consider a membrane in which all pumps are continuously active 


\section{Sriram Ramaswamy, John Toner and Jacques Prost}

when supplied with energy; allowing the pumps to switch on and off at random modifies our results in a manner irrelevant at small wavenumbers [14].

Let the membrane conformation with respect to a two-dimensional reference coordinate $\mathbf{x}$ at time $t$ be described within the Monge gauge by a height function $h(\mathbf{x}, t)$, and let $n_{+}(\mathbf{x}, t)$ and $n_{-}(\mathbf{x}, t)$ be the local concentrations of + and - pumps respectively. Define the protein density (irrespective of whether they are + or - )

$$
n(\mathbf{x}, t) \equiv n_{+}(\mathbf{x}, t)+n_{-}(\mathbf{x}, t)
$$

and the pump density (the relative number density of + and - pumps)

$$
m(\mathbf{x}, t) \equiv n_{+}(\mathbf{x}, t)-n_{-}(\mathbf{x}, t)
$$

with time-averages $n_{0}$ and $m_{0}$ respectively. Since $n$ plays an unimportant role in what follows, we shall ignore its dynamics hereafter, setting its value to a constant $n_{0}$. Let us first consider the case of a membrane at equilibrium, where the proteins are present but not activated. As remarked in $\S 1$, the proteins will in general have an asymmetric shape, so that + and - proteins will favour opposite signs of the local mean curvature (see figure 2).

The effective Hamiltonian describing the equilibrium statistical mechanics of the membrane with such asymmetric but inactive proteins, to bilinear order in the variables involved, is thus

$$
H[m, h]=\frac{1}{2} \int \mathrm{d}^{2} x\left[\kappa\left(\nabla^{2} h\right)^{2}+\chi^{-1}\left(m-m_{0}\right)^{2}+T \ell m \nabla^{2} h\right],
$$

a form studied in [13] in connection with phase separation in membranes. The energy density in (3) has three terms. The first is the usual mean-curvature energy [3], with rigidity $\kappa$. The second is the compression energy for the signed protein number density, with osmotic modulus $\chi^{-1}$. For convenience, we have separated out a factor of the temperature $T$ in the third term, which couples the signed pump density to the local mean curvature. With this convention, the coefficient $\ell$ in (3) has dimensions of a length. Its magnitude may be interpreted tentatively as the head-tail size difference of a protein, but its sign has a siginficance for the dynamics in the active state, as we shall see below. The equations of motion for the membrane at equilibrium, which we do not list separately here, follow from the simple "Rouse" assumption, in which the normal velocity of the membrane is proportional to the total normal force $\delta H / \delta h(\mathbf{x}, t)$ on the membrane, and Fick's law, i.e. the current of $m$ is proportional to the chemical potential gradient $\nabla \delta H / \delta m(\mathbf{x}, t)$.

The active state, in which the proteins are given a steady supply of energy, differs qualitatively from the above equilibrium state. Several terms of nonequilibrium origin enter the equations of motion, with a form constrained by the symmetry $h \rightarrow-h$, $m \rightarrow-m$. To understand these systematically it is useful to distinguish, as we did in section 1.1 , the balanced $\left(m_{0}=0\right)$ and unbalanced $\left(m_{0} \neq 0\right)$ cases. The balanced case is the simplest, and is appropriate for experiments such as those of [10] on large membranes in which there is little control on the orientation of the implanted proteins. This means in particular that the mean normal drift speed of the membrane on macroscopic scales is zero. Many of the nonequilibrium terms are nonlinear, involving products of $m(\mathbf{x}, t)$ with itself or with derivatives of the height field. Many of our results, however, are obtained within a strictly linearised description of fluctuations in the balanced case $m_{0}=0$. Away 


\section{Membranes with active pumps}

from this simplifying but physically relevant limit, additional nonequilibrium terms arise which we shall discuss in detail elsewhere [14].

In the linearised $m_{0}=0$ limit, the membrane height field satisfies

$$
\begin{aligned}
\frac{\partial h}{\partial t} & =\lambda_{p}\left(-\delta H / \delta h+F_{a} m\right)+f_{h} \\
& =\lambda_{p}\left(-\kappa \nabla^{4} h-T \ell \nabla^{2} m+F_{a} m\right)+f_{h}
\end{aligned}
$$

and the pump density obeys the conservation law

$$
\begin{aligned}
\frac{\partial m}{\partial t} & =\Lambda \nabla^{2} \delta H / \delta m+\nabla \cdot \mathbf{f}_{m} \\
& =\Lambda \chi^{-1} \nabla^{2} m-\Lambda T \ell \nabla^{4} h+\nabla \cdot \mathbf{f}_{m}
\end{aligned}
$$

with $H$ given by (3). Equation (4) says that the vertical speed of the membrane is given by a mobility $\left(\lambda_{p}\right.$, the permeation kinetic coefficient) times the areal force density. The latter, in parentheses on the first line of (4), has two pieces. The first is the elastic restoring force of the membrane, and the second the force density arising from the protein activity. Each active protein exerts a force $F_{a}$ normal to the membrane. A pump density $m(\mathbf{x}, t)$ thus yields a force density $F_{a} m(\mathbf{x}, t)$, neglecting for simplicity any dependence of the activity of a given protein on the presence of other proteins. The head-tail asymmetry of a pump allows its activity to depend on the local mean curvature, so that (4) should contain an additional nonequilibrium term proportional to $\nabla^{2} h$. Such a term is unimportant to the discussion below; its effects, in particular an instability if the term has a negative coefficient, will be presented elsewhere [12].

Equation (5) at this level of description is precisely as it would be at equilibrium: the pump density diffuses in response to chemical potential gradients, with a mobility $\Lambda$. Equations (4) and (5) contain spatiotemporally white noise sources $f_{h}$ and $\mathbf{f}_{m}$ respectively. At thermal equilibrium, these have variance proportional to $\lambda_{p}$ and $\Lambda$ respectively. We will retain this form in the active state as well, since the dissipative processes that gave rise to it are still present; we ignore for now the possibility of additional sources of noise.

In (4) and (5), we take $F_{a}>0$ without loss of generality. All other parameters are positive, except $\ell$, the phenomenological coupling between curvature and pump density. For $\ell<0$, the equations are linearly unstable at small wavenumber. We defer consideration of this interesting possibility to a later publication [14], and assume $\ell>0$ in the treatment below.

For $\ell>0$, (4) and (5) lead to a stable nonequilibrium steady state, with a height variance $\left\langle\left|h_{k}\right|^{2}\right\rangle \sim 1 / k^{4}$ for wavenumber $k \rightarrow 0$, with a coefficient independent of $\kappa$. To see this, Fourier-transform (4) and (5) in space and time, invert the resulting linear system to yield the fourier-transformed fields $h_{\mathbf{k} \omega}$ and $m_{\mathbf{k} \omega}$ for wavevector $\mathbf{k}$ and frequency $\omega$ in terms of the noise sources, and calculate $\left\langle\left|h_{\mathbf{k} \omega}\right|^{2}\right\rangle$ and $\left\langle\left|m_{\mathbf{k} \omega}\right|^{2}\right\rangle$ using the statistical properties of the noise. Then

$$
\begin{aligned}
\left\langle\left|h_{k}\right|^{2}\right\rangle & =\int \frac{\mathrm{d} \omega}{2 \pi}\left\langle\left|h_{\mathbf{k} \omega}\right|^{2}\right\rangle \\
& \sim \frac{\lambda_{p} F_{a}}{\Lambda \chi^{-1} \ell k^{4}} .
\end{aligned}
$$




\section{Sriram Ramaswamy, John Toner and Jacques Prost}

The behaviour predicted by (6), namely, variance scaling as $1 / k^{4}$ and depending neither on the bending modulus nor on the number density of active proteins, is precisely as seen in experiments by Manneville et al [10]. The crossover to the equilibrium form $T / \kappa k^{4}$ is subtle and takes place after passage through an intermediate $k^{-6}$ regime (the analogue in this "Rouse" model of the $k^{-5}$ predicted in [8] in a treatment ignoring the curvatureconcentration coupling $\ell$ of (3)).

\section{Conclusion}

Working to strictly linear order in the dynamical fields, and assuming a linearly stable, "balanced" membrane, we have arrived at the startling prediction that an active membrane can display nonequilibrium height fluctuations whose wavenumber dependence can mimic that of an equilibrium membrane. We believe this is the explanation of the observations of [10], and we urge further experiments to test our predictions. Departures from the simplifying assumptions of this paper lead to further interesting predictions, including propagating modes and nontrivial scaling exponents. In addition, we have merely mentioned the possible mechanisms for instability in active membranes. A complete treatment of these intriguing effects, including the asymptotic long-time behaviour in the linearly unstable case, will appear elswehere [14-16].

\section{Acknowledgements}

We thank P B Sunil Kumar, R Nityananda, A Pande, R Pandit, and M Rao for valuable discussions, and A Guha for help with figures.

\section{References}

[1] H Lodish et al, Molecular Cell Biology, 3rd edition (W H Freeman, New York, 1995)

[2] M D Houslay and K K Stanley, Dynamics of Biological Membranes (Wiley, New York, 1982)

[3] P B Canham, J. Theor. Biol. 26, 61-81 (1970)

W Helfrich, Z Naturforsch. C28, 693-703 (1973)

[4] U Seifert, Adv. Phys. 46, 13-137 (1997)

[5] S Levin and R Korenstein, Biophys. J. 60, 733-737 (1991)

[6] S Tuvia et al, Proc. Natl. Acad. Sci. U.S.A. 94, 5045-5049 (1997)

[7] F Brochard and J-F Lennon, J. Phys. 36, 1035-1047 (1975)

[8] J Prost and R Bruinsma, Europhys. Lett. 33, 321-326 (1996)

[9] J Prost, J-B Manneville and R Bruinsma, Eur. Phys. J. B1, 465-481 (1998)

[10] J B Manneville, P Bassereau, D Lévy and J Prost, Phys. Rev. Lett. 82, 4356-4359 (1999)

[11] More precisely, it means that we cannot on symmetry grounds rule out such a structural asymmetry and must therefore allow for it

[12] This instability is independent of the equilibrium instability of two-component membranes [11]

[13] D Andelman, T Kawakatsu and K Kawakatsu, Europhys. Lett. 19, 57 (1992)

P B Sunil Kumar and M Rao, Phys. Rev. Lett. 80, 2489-2492 (1998)

[14] S Ramaswamy, J Toner and J Prost (in preparation)

[15] A Pande R Pandit and S Ramaswamy (unpublished)

[16] P B Sunil Kumar, M Rao and S Ramaswamy (unpublished)

[17] M Doi and S F Edwards, The Theory of Polymer Dynamics (Clarendon, Oxford, 1986) 